This item was submitted to Loughborough's Research Repository by the author.

Items in Figshare are protected by copyright, with all rights reserved, unless otherwise indicated.

\title{
Neutral daylight illumination with variable transmission glass: theory and validation
}

PLEASE CITE THE PUBLISHED VERSION

http://dx.doi.org/10.1177/1477153515620339

PUBLISHER

(c) SAGE Publications

VERSION

AM (Accepted Manuscript)

\section{PUBLISHER STATEMENT}

This work is made available according to the conditions of the Creative Commons Attribution-NonCommercialNoDerivatives 4.0 International (CC BY-NC-ND 4.0) licence. Full details of this licence are available at: https://creativecommons.org/licenses/by-nc-nd/4.0/

\section{LICENCE}

CC BY-NC-ND 4.0

\section{REPOSITORY RECORD}

Mardaljevic, John, Birgit Painter, and Ruth Kelly Waskett. 2015. "Neutral Daylight Illumination with Variable Transmission Glass: Theory and Validation". Loughborough University. https://hdl.handle.net/2134/18140. 


\section{Neutral daylight illumination with variable transmission glass: theory and validation}

J Mardaljevic PhD FSLL ${ }^{a}$, R Kelly Waskett ${ }^{b}$ and B Painter $\mathrm{PhD}^{b}$

${ }^{a}$ School of Civil and Building Engineering, Loughborough University, Loughborough, UK

${ }^{b}$ Institute of Energy and Sustainable Development, De Montfort University, Leicester, UK

Address for correspondence: Professor John Mardaljevic, School of Civil and Building Engineering, Loughborough University,

Loughborough, Leicestershire, LE11 3TU, UK

E-mail: j.mardaljevic@lboro.ac.uk

Received XX XXX 2014; Revised XX XXX 2015; Accepted XX XXX 2015 
The dynamic control of daylight is seen as key for the effective exploitation of natural illumination in buildings. Traditional control solutions are invariably used in a suboptimal manner: blinds/shades are left down for long periods and lights are left switched on. A glazing with a transmissivity that varies continuously between clear and dark extremes, and which can be controlled automatically, could be much more effective in providing a 'well-tempered' daylit environment that meets occupants needs. Amongst the different types of variable transmission glazing that have undergone extensive research and development in the last few decades, those based on electrochromism appear to have the best performance characteristics and the greatest market potential. Electrochromic glazing generally exhibits a shift in spectral transmission as the glass darkens, e.g. causing it to appear blue as it tints. Occupants however are believed to prefer a neutral spectrum of daylight illumination without any pronounced hue. In this paper the authors show that it is possible to maintain a neutral spectrum of illumination with EC glazing under normal operation provided that just a small proportion of the EC glazing is kept in the clear state. A theoretical model to predict the daylight spectrum resulting from any arbitrary combination of clear and tinted glazing is described. Predictions from the model are compared with measurements of the daylight spectra in an office with EC glazing under various states of tint. The predicted spectra show excellent agreement with the measurements. The model is applicable to any combination of clear and/or tinted glass panels irrespective of the glazing type(s). The paper concludes with a discussion of design considerations for the effective deployment of EC glazing.

\section{Introduction}

The use of daylight in office buildings is generally considered to be a greatly underexploited resource. In large part this is because of the highly variable nature of daylight illumination. The natural, large variability in daylight means that users will often need to use shades to moderate excessive ingress of daylight. Most shading systems are often operated as a "shutter" that is either open or closed, with users rarely making the effort to optimise the shading for both daylight provision and solar/glare control. For example, venetian blinds can be used to both redirect light and offer protection from direct sun. However, effective operation/adjustment of venetian or slatted blinds is probably the exception rather than the rule. Also, blinds are often left closed long after the external condition has changed. A glazing with a transmissivity that varies continuously between clear and dark extremes could offer a much greater degree of control over the luminous environment.

The principle behind variable transmission glazing (VTG) is straightforward: the transmission properties of the glazing are varied to achieve an 'optimum' luminous and/or thermal environment. The various types of VTG can be grouped into three broad classes: chromogenic, suspended particle device and micro-electromechanical systems. In the chromogenic class there are four distinct types of formulations that have variable transmission properties. These are: electrochromic, gasochromic, photochromic and thermochromic. The agents causing the change in transmission are: voltage (electrochromic); concentration of pumped gas (gasochromic); localised illumination (photochromic); and, localised temperature (thermochromic). Thermochromic and photochromic are essentially passive devices which respond to changes in the environment, whereas electrochromic and gasochromic are active devices that can be configured to respond to any sensor input, e.g. illumination, temperature, or some combination of the two. Suspended particle device 
(SPD) is a plastic PET film-based technology. The plastic film contains a suspension of rod-like particles in billions of liquid droplets. An applied voltage alters the orientation of the particles and therefore the transmission properties of the film. A VTG based on micro-electromechanical systems (MEMS) has tiny, micron-scale structures that move in response to an applied electrostatic field, thereby altering the transmission properties of the glazing.

The key to performance for a VTG is a high (visible) transmission in the clear state and a sufficiently low (visible) transmission in the darkened (or tinted) state. To be perceived as acceptable to the majority of building occupants, the VTG in the clear state should appear like ordinary (un-tinted) double glazing, and so have a visible transmission of around $60 \%$ or greater. In the darkened state the transmission should be low enough so that additional shading is required only very rarely, or perhaps not at all. In practice this means a minimum visible transmission of around $2 \%$ or less - a feat achieved for production electrochromic glazing. Additionally, the building occupants should have some degree of control of the glazing, e.g. to manually override an automated control setting. Experience has shown that occupants will often resort to sabotage if an automated building control system fails to do what they wish. So, whilst a 'passive' VTG might seem attractive at first because it allows for autonomous operating behaviour, the corollary of this is a lack of control, e.g. modulation of the glazing transmission by (localised) window temperature will not necessarily offer the luminous environment desired by the occupants.

There are examples of thermochromic glazing on the market, though the narrow visible transmission range (e.g. 13-60\% or 6-30\%) indicates that additional shading would be needed to control glare. Thermochromic glazing therefore seems better suited to offering a degree of moderation of the thermal rather than the luminous environment. Gasochromic has the potential advantage of rapid switching speeds. A gasochromic system requires that the glazing unit is literally 'plumbed-in' - connected to an electrolyser and pump by piping. The practicalities of a gasochromic installation are such that the technology is still considered the preserve of research. Suspended particle device for clear (i.e. view) windows appears to be still 'near-to-market' rather than market ready, and MEMS glazing technology seems to be still undergoing development. Thus, of the technologies described above, only electrochromic (EC) glazing appears to have the necessary optical properties (i.e. wide visible transmission range), is relatively straightforward to install, and is already in the marketplace.

\subsection{Electrochromic glazing}

Electrochromism has been known since the 19th Century [1], and its application as a glazing technology has been investigated since the 1980s [2]. However, it is only recently with major new investment and scaled-up production that EC glazing has shown the potential to become a mainstream product [3]. In effect, EC glass mimics the function of the iris in the mammalian eye by varying the overall transmission whilst maintaing a clear view. EC glass is therefore an example of "bio-mimicry" in architectural design an appreciation that appears to have been overlooked in the past.

The effectiveness of EC glazing to temper the indoor thermal environment has been demonstrated in a number of theoretical and empirical studies [4], and modelling its performance in a dynamic thermal simulation program is relatively straightforward [5]. It is however the user acceptance of the luminous environment produced by EC glazing that will be the key determinant for the success of this VTG technology [6].

User acceptance for any daylight control technology depends on a number of perfor- 
mance and operational characteristics. For EC glazing these include performance with respect to glazing transmission range (i.e. the values for the maximum and minimum visible transmittances), the switching time between the clear and tinted states and the effectiveness of the automated control to minimise user interventions (e.g. manual overrides). Another key factor for user acceptance is the quality of the luminous environment produced by EC glazing. An important component of this is the spectral composition of the daylight that is 'filtered' through tinted EC glass This is because the spectral transmission properties of the EC coating varies as the glass changes state. This can be seen in Figure 1 showing a pair of photographs with EC glass in the clear state (left) and at full-tint (right). As the glass darkens (i.e. 'tints') the longer wavelengths are diminished proportionally to a greater degree than the shorter wavelengths, giving the EC glazing good solar control properties to help prevent overheating. Optically, the consequence of this is to shift the peak in visible transmission to the blue end of the spectrum.
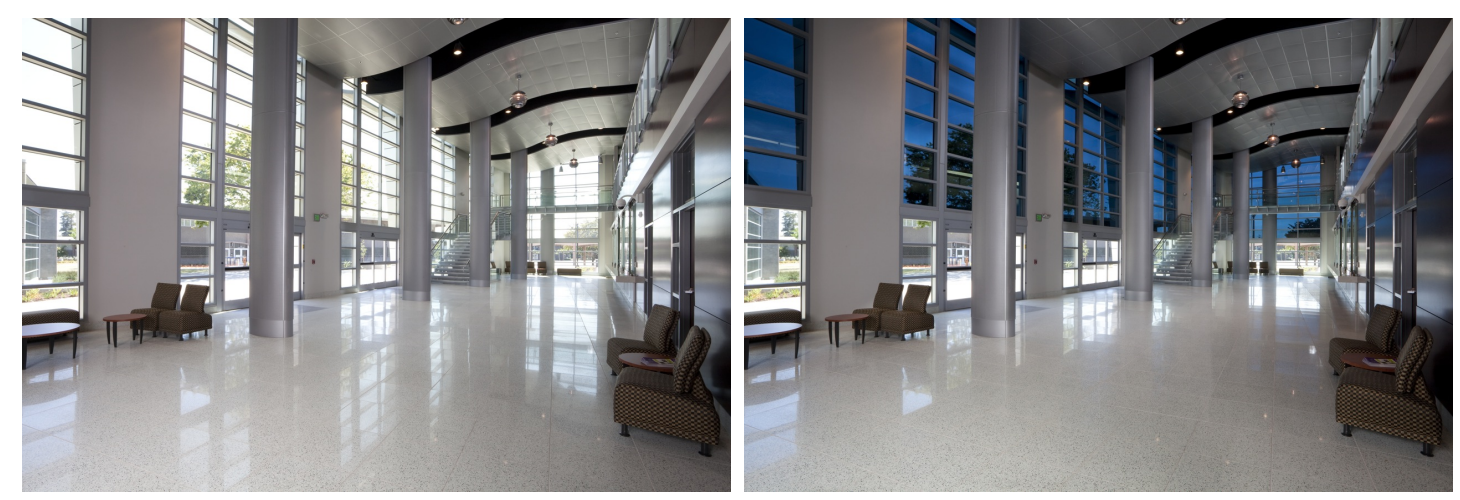

Figure 1: Images showing electrochromic glazing in clear and darkened state (photos courtesy SAGE Electrochromics Inc.)

This can be seen in the transmission curves for SageGlass EC glazing shown in Figure 2. In the clear state the EC glazing has a visible transmittance of $62 \%$ and appears effectively neutral to the eye. There is a slight 'peak' in the curve around $600 \mathrm{mn}$ giving a very slight straw coloured hue, though this is generally not noticeable in normal use. This product has a minimum visible transmittance of $2 \%$ when fully tinted and can be varied continuously between this and the clear state. However a small number of intermediate states is considered adequate for most practical installations, e.g. 'light-tint' (20\%) and 'mid-tint' (6\%). Note: the transmission curves are those for SageGlass EC glazing manufactured in 2012 and installed in the offices used for the validation described below. The current generation of SageGlass varies in visible transmission between $60 \%$ (clear) and 1\% (fullytinted). The findings shown below are equally applicable to the current product.

The peak in the spectral transmission curves gradually shifts from $615 \mathrm{~nm}$ in the clear state to $455 \mathrm{~nm}$ at full-tint. Thus the view through the glazing takes on a progressively deeper blue hue as it transitions from clear to full-tint (Figure 1). And of course, the daylight transmitted through the window will be 'filtered' according to the spectral properties of the glazing and the character of the illumination incident on the glazing, e.g. 'warm' sunlight, 'blue' skylight, etc. This presents a number of potential user acceptance issues for any EC glazing installation. In particular, ensuring that the daylight illumination in the space is perceived as 'neutral' and adequate for everyday colour rendering purposes. There have been reports that 'blue' fixed-tinted glazing had lower approval ratings from 
test subjects than neutral or warm fixed-tinted glass [7]. Thus the question regarding the neutrality of the illumination spectrum is an important one that needs to be addressed.

In this study the authors demonstrate that it is possible to maintain an effectively neutral spectrum of daylight illumination in a space with EC glass in normal operation, provided that a relatively small proportion of the glass is left in the clear state. We present a theoretical formulation giving the overall spectral transmittance curves for any arbitrary combination of clear and tinted EC glazing in varying proportions. Applying the theoretical model it should be possible to configure and/or control an actual EC glass installation so that neutral daylight illumination results, even during times when a high degree of daylight/solar control is required. The theoretical model is tested using measurements of the daylight spectra in an office space with EC glazing for six combinations of clear and tinted glass. The paper concludes with:

(a) a discussion on the design of facades with EC glass to ensure that the neutral illumination spectra predicted by the model is achieved; and,

(b) two hypotheses regarding occupant approval of EC glass.

The following section describes the theoretical model.

\section{Theoretical model}

A general matrix formulation to determine the overall spectral transmittance curve for any arbitrary combination of EC glass with known transmission spectra is presented. Following the mathematical description an example is given based on spectral transmittance data for a commercial EC glazing product manufactured by SAGE Glass (Minnesota, USA). The spectral data for the EC glass in each of the four commonly used states of tint were obtained from the respective International Glazing Database Files. The spectral transmittance data covers the range 300 to $2,500 \mathrm{~nm}$ in increments of $5 \mathrm{~nm}$ (Figure 2).

\subsection{Matrix formulation}

The procedure to derive the overall transmittance curve for an arbitrary combination of EC panels in various states is as follows. The row vector $\mathbf{V}$ of the visible transmittance of the glazing in four possible states of tint $a, b, c$ and $d$ is:

$$
\mathbf{V}=\left[\begin{array}{llll}
V_{a} & V_{b} & V_{c} & V_{d}
\end{array}\right]
$$

In the commonly used states this vector would equal $\left[\begin{array}{llll}0.62 & 0.20 & 0.06 & 0.02\end{array}\right]$, i.e. $62 \%$ visible transmittance in the clear state, $2 \%$ when fully tinted, and the two intermediate states of $20 \%$ and $6 \%$. Next, the row vector $\mathbf{R}$ gives the ratio or number of equal-sized panels in each of the states of tint:

$$
\mathbf{R}=\left[\begin{array}{llll}
N_{a} & N_{b} & N_{c} & N_{d}
\end{array}\right]
$$

For example, if one panel was in the clear state, two at light-tint, three at mid-tint and four at full-tint, the vector would equal $\left[\begin{array}{llll}1 & 2 & 3 & 4\end{array}\right]$.

The effective visible transmittance of the combination $V_{R}$ is determined from the following equation:

$$
V_{R}=\frac{\left[\begin{array}{llll}
V_{a} & V_{b} & V_{c} & V_{d}
\end{array}\right] \cdot\left[\begin{array}{llll}
N_{a} & N_{b} & N_{c} & N_{d}
\end{array}\right]^{\top}}{\left(N_{a}+N_{b}+N_{c}+N_{d}\right)}
$$



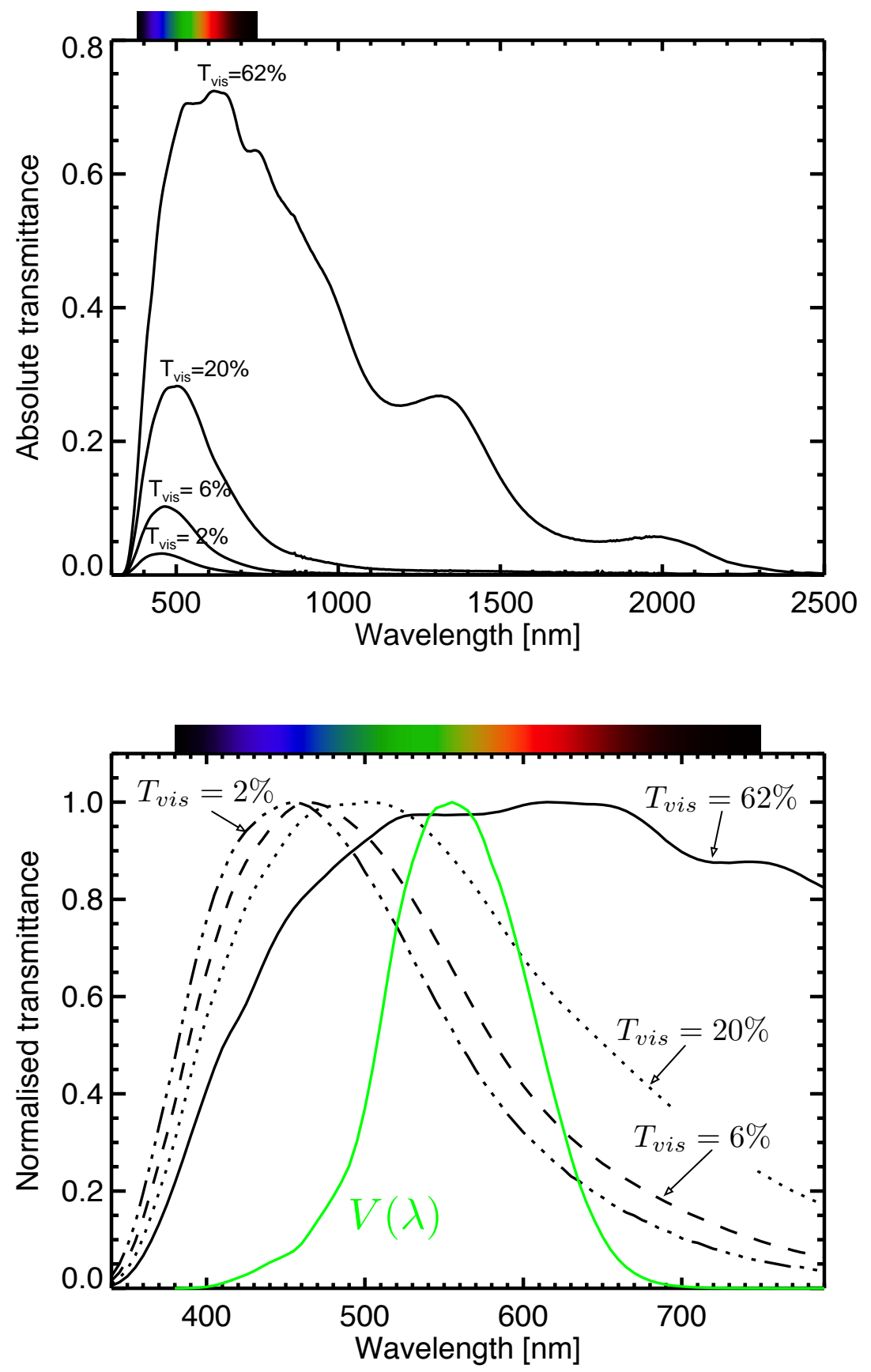

Figure 2: Absolute and normalised spectral transmission curves for SAGE electrochromic glass in clear $(62 \%)$, fully tinted $(2 \%)$ and two intermediate states. Data from the IGDB files supplied by SAGE Glass. The normalised plot also includes the visual sensitivity curve $V(\lambda)$. 
where $^{\top}$ denotes the transpose from row to column vector. The same more compactly is:

$$
V_{R}=\frac{\mathbf{V} \cdot \mathbf{R}^{\top}}{\sum \mathbf{R}}
$$

Of particular interest for this study is the fraction of the total transmittance that is from glass in the clear state (i.e. where $V_{a}=0.62$ ) since this will be a key determinant in achieving neutral daylight illumination inside the space. The fraction $P_{a}$ is determined as follows:

$$
P_{a}=\frac{V_{a} R_{a}}{\sum_{i=1}^{4} V_{i} R_{i}}
$$

The overall spectral transmittance curve for any arbitrary combination of (equal-sized) EC panels is determined using similar matrix operations. For example, the vector $\mathbf{T}_{\mathbf{a}}$ of spectral transmittance data for EC glass in state $a$ is given by:

$$
\mathbf{T}_{\mathbf{a}}=\left[\begin{array}{llll}
t_{a_{1}} & t_{a_{2}} & \cdots & t_{a_{m}}
\end{array}\right]
$$

where, for the IGDB data used here, $t_{a_{1}}$ is the transmittance at $300 \mathrm{~nm}$ and $t_{a_{m}}$ the transmittance at $2,500 \mathrm{~nm}$. The four individual row vectors comprising the four spectral transmittance curves shown in Figure 2 are concatenated to form a matrix:

$$
\left[\begin{array}{llll}
t_{a_{1}} & t_{a_{2}} & \cdots & t_{a_{m}} \\
t_{b_{1}} & t_{b_{2}} & \cdots & t_{b_{m}} \\
t_{c_{1}} & t_{c_{2}} & \cdots & t_{c_{m}} \\
t_{d_{1}} & t_{d_{2}} & \cdots & t_{d_{m}}
\end{array}\right] \quad \text { or } \quad\left[\begin{array}{c}
\mathbf{T}_{a} \\
\mathbf{T}_{b} \\
\mathbf{T}_{c} \\
\mathbf{T}_{d}
\end{array}\right]
$$

The vector $\mathbf{T}_{\mathbf{R}}$ containing the spectral transmittance curve data for an arbitrary combination $\mathbf{R}$ of EC panels in various states is given by:

$$
\mathbf{T}_{\mathbf{R}}=\left[\begin{array}{llll}
N_{a} & N_{b} & N_{c} & N_{d}
\end{array}\right] \cdot\left[\begin{array}{llll}
t_{a_{1}} & t_{a_{2}} & \cdots & t_{a_{m}} \\
t_{b_{1}} & t_{b_{2}} & \cdots & t_{b_{m}} \\
t_{c_{1}} & t_{c_{2}} & \cdots & t_{c_{m}} \\
t_{d_{1}} & t_{d_{2}} & \cdots & t_{d_{m}}
\end{array}\right] /\left(N_{a}+N_{b}+N_{c}+N_{d}\right)
$$

Or, more compactly:

$$
\mathbf{T}_{\mathbf{R}}=\frac{\mathbf{R} \cdot\left[\begin{array}{llll}
\mathbf{T}_{a} & \mathbf{T}_{b} & \mathbf{T}_{c} & \mathbf{T}_{d}
\end{array}\right]^{\top}}{\sum \mathbf{R}}
$$

This equation can be used to derive the combined spectral transmittance curve for arbitrary combinations of (equal-sized) EC panels in various states. For panes of dissimilar sizes, an additional (vector) term needs to be incorporated into the above equations to weight the individual contributions to the combination curve accordingly.

\section{$2.2 \quad$ Predicted properties}

With even a small number of independently controlled EC panels there are many unique combinations of tint state possible. Initial tests showed that to achieve high levels of overall daylight control, whilst maintaining at the same time the potential for neutral daylight illumination, a combination of clear and fully tinted panels is particularly effective. For example, a two panel combination with one set to fully clear $(62 \%)$ and one to full-tint $(2 \%)$ has an equivalent visible transmittance of $32 \%$, i.e. $(62+2) / 2$. However, 
approximately $97 \%$ of that equivalent visible transmittance is due to the panel in the clear state, because of course the clear state panel has $31 \times$ the visible transmittance of the glass at full-tint. Below are compared the overall spectra for one clear panel with up to eight panels at full-tint (with none set to either of the intermediate states):

$$
\mathbf{R}=\left[\begin{array}{llll}
1 & 0 & 0 & N_{d}
\end{array}\right] \text { where } N_{d}=1,2,3,4,5,6,7 \text { or } 8
$$

For each of the eight values of $N_{d}$ in $\mathbf{R}$, Equation (7) was used to determine the spectral transmittance curve $\mathbf{T}_{\mathbf{R}}$ of the combination.

Any comparison of spectral transmittance curves will depend to a degree on how the data are normalised. The combination spectral curves (Figure 3) are shown each normalised to peak value equals 1 because, for this part of the evaluation, it the the shape of the spectra rather than their absolute values which are of importance. Additionally, the plot shows the transmittance curves for EC glass in the clear state (dashed line) and at full-tint (dotted line), Figure 3. For reference, the plot also includes the visual sensitivity curve $V(\lambda)$ (also normalised to peak equals 1 ).

The curve for $\mathbf{R}=\left[\begin{array}{llll}1 & 0 & 0 & 1\end{array}\right]$ (i.e. 1 clear panel and 1 at full-tint) is shown in yellow. With gradually increasing values for $N_{d}$, the colour used for the curve transitions from yellow through red to blue, i.e. for $\mathbf{R}=\left[\begin{array}{llll}1 & 0 & 0 & 8\end{array}\right]$. Although containing many curves, the plot is quite easy to interpret when viewed in colour since the progression with increasing number of full-tint panels is quite pronounced and 'orderly'. Firstly, for the case with 1 clear and 1 full-tint panel, the combined spectral transmittance curve (yellow) is almost identical to that for the glass in the clear sate (dashed line). Relative to the clear state, there is a very slight suppression of wavelengths longer than $500 \mathrm{~nm}$ (i.e. the 'red' end of the visible spectrum), and a slight enhancement of wavelengths shorter than $500 \mathrm{~nm}$ (i.e. the 'blue' end). With each additional full-tint panel this trend persists, with the crossover point around $500 \mathrm{~nm}$ seeming to act as a 'pivot'.

Even with eight full-tint panels for every one that was clear, the (normalised) transmittance curve for the combination is, qualitatively, much closer to the curve for the clear state than that for the full-tint state. Note also that, with reference to the visual sensitivity curve $V(\lambda)$, all eight curves are fairly 'flat'. This is better appreciated in the additional set of (coloured) curves where now the spectral transmittance value at each wavelength is shown relative to that for the glass in the clear state. These curves are all in the lower half of the plot. For example, the transmittance values for $\mathbf{R}=\left[\begin{array}{llll}1 & 0 & 0 & 1\end{array}\right]$ (yellow curve) are approximately half those of the corresponding values for the clear state, since, in that combination, the overall visible transmittance is about half that for EC glass in the clear state (dashed line).

The predicted performance of the combinations are summarised in Figure 4. Here the effective visible transmittance for each combination (equation 2) and the percentage of the total transmittance from glass in the clear state (equation 3 ) for $N_{d}$ equals 0 to 8 are plotted using the same percentage scale. Also shown is the percentage of the total transmittance resulting from glass panels at full-tint. With $N_{d}=8$ the effective visible transmittance of the combination is just $8.7 \%$, but nevertheless $79.5 \%$ of the combined visible transmittance is due to the glass in the clear state. Hence, even with this arrangement one might reasonably expect the illumination to be fairly neutral, i.e. fairly 'flat' across the visible range. This hypothesis is tested in the validation section.

\subsection{The illumination spectrum}

The procedure described above predicts the transmittance spectrum for an arbitrary combination of EC glass in various states. The illumination spectrum - that is, the daylight 


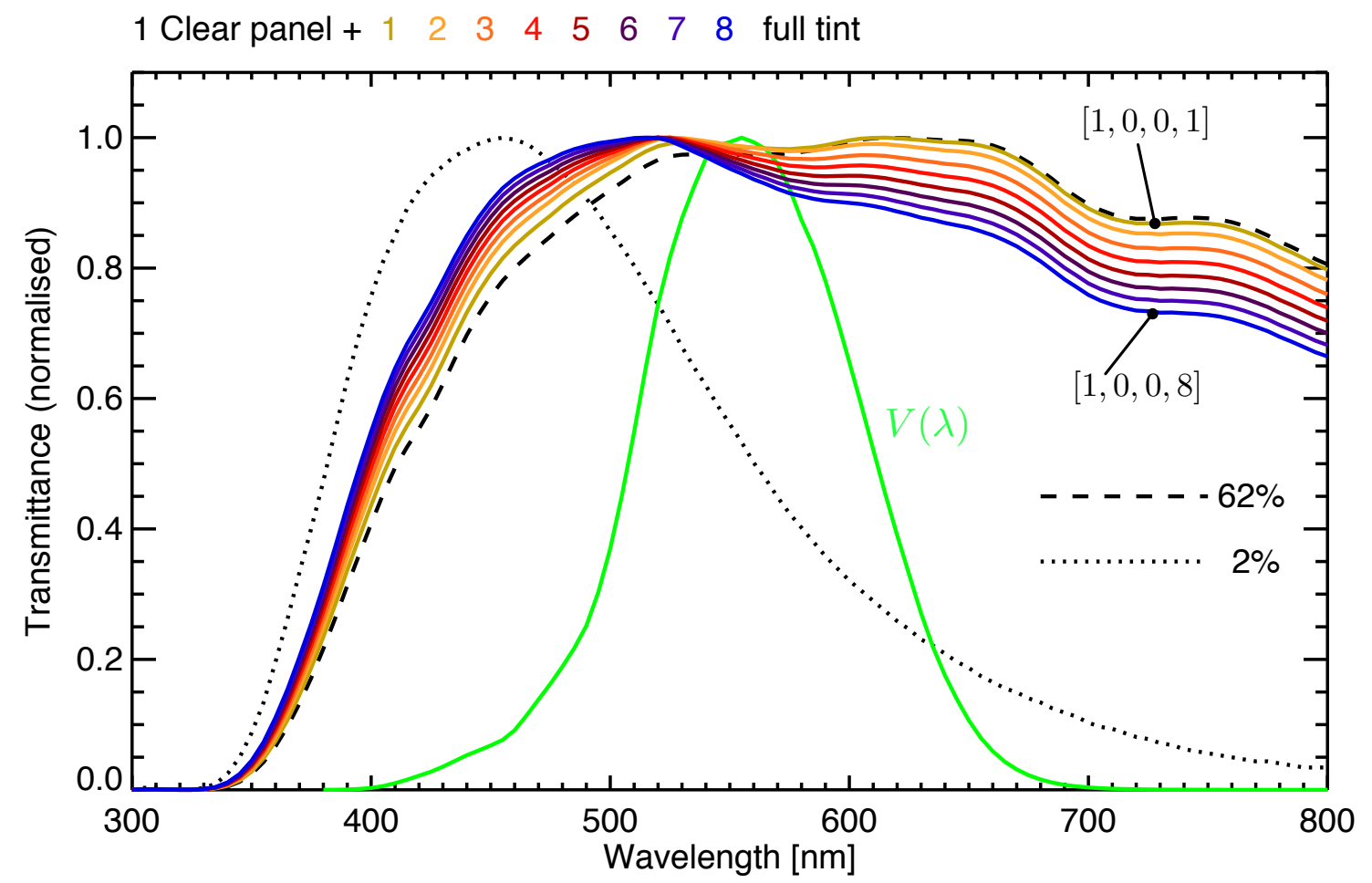

Figure 3: Eight spectral transmittance curves for EC glass in the combinations given in Equation (8).

that passes through the glazing to illuminate the space - will depend also on the spectrum of light that is incident on the glass. The illumination spectrum is given by the vector $\mathbf{I}_{\mathbf{T}_{\mathbf{R}}, \mathbf{D}_{\mathbf{K}}}$ where $\mathbf{T}_{\mathbf{R}}$ refers to transmittance spectrum produced by $\mathrm{EC}$ combination $\mathbf{R}$, and $\mathbf{D}_{\mathbf{K}}$ is the vector containing the spectral power distribution for one of the standard daylight illuminants, e.g. $D_{55}, D_{65}$, etc. The illumination spectrum is given by:

$$
\mathbf{I}_{\mathbf{T}_{\mathbf{R}}, \mathbf{D}_{\mathrm{K}}}=\mathbf{T}_{\mathbf{R}} \circ \mathbf{D}_{\mathrm{K}}
$$

Where $\circ$ is the symbol for the element-by-element or Hadamard product. For any pair of matrices (or vectors) $\mathbf{A}$ and $\mathbf{B}$ of equal dimensions, the Hadamard product $\mathbf{C}=$ $\mathbf{A} \circ \mathbf{B}$, where each element $C_{i}=A_{i} B_{i}$ (for a 1-dimensional matrix). This is illustrated schematically in Figure 5.

\section{Validation}

The theoretical schema described above was tested in an office space containing eight panels of SAGE electrochromic glass which have the spectral characteristics shown in Figure 2. The validation scenario is described in the following section.

\subsection{The electrochromic glazing installation}

Although EC glass has been available for a number of years and evaluated under various experimental conditions (e.g. test cells), the first commercial installation in the UK happened only in late 2012. Two offices at De Montfort University (Leicester, UK) were fitted with EC glazing produced by SAGE Electrochromics Inc. The lighting in the offices was upgraded at the same time, but otherwise the offices and the occupants were as 


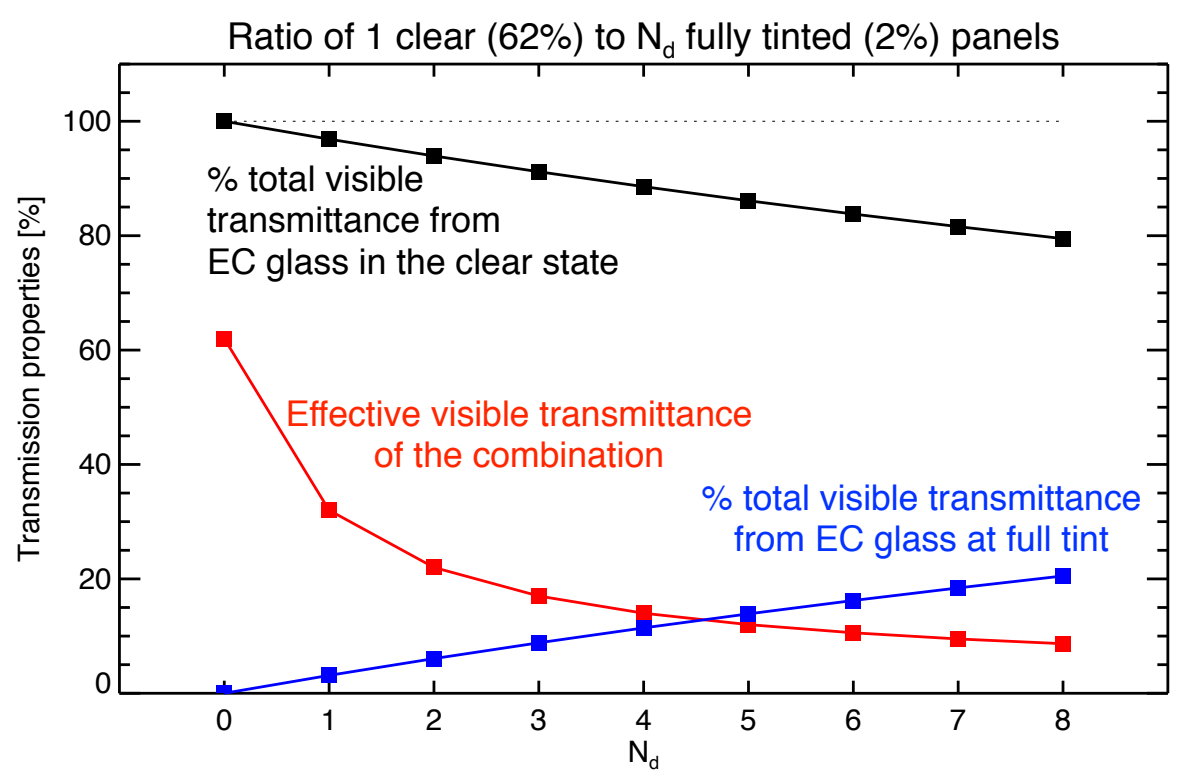

Figure 4: Summary of transmission properties for one clear panel and up to eight at full-tint.

before. The user acceptance of the installation is being evaluated as part of a long-term case study [8]. In addition to being the first office building in the UK with EC glass, the case-study evaluation is perhaps unique precisely because it is such an ordinary office space with occupants going about their everyday tasks. In contrast, many of the other EC installations to date have been large transit spaces (e.g. Figure 1) or conference rooms with intermittent occupancy and limited scope to capture 'real world' user experience under typical working conditions [6].

Photographs of the office that was used for this field-study (Room 0.30) and the external facade are given in Figure 6. Note that the installation was a non-standard retrofit. The two offices (Rooms 0.30 and 0.29 ) comprise three large window bays, each with six panels. However, the dividing wall between the two offices bisects the central bay. Additionally, the false ceiling in the offices meets the facade wall at the shared window and the window exclusive to 0.29. Thus the upper panels for these two bays are either for ventilation or are 'false' windows, i.e. they do not provide any illumination to the offices. For the remaining bay in 0.30 , the false ceiling is stepped back from the window, and all six panels can illuminate the space - though the false ceiling does offer some shading depending on the sun angle. Thus, there are eight EC panels in room 0.30 and six in room 0.29 - they were all set to full-tint when the external photograph was taken (Figure 6).

\subsection{Glazing states tested}

The zoning arrangement used to control the glazing in 0.30 is shown in Figure 7 (zones 1-4 were assigned to the the four zones in Room 0.29). In the full-height six pane window, each row-pair of panels constitutes a zone - thus there are five zones in total. The 'housekeeping' label used to describe a particular state for the EC glass in Room 0.30 is a series of five numbers each between 1 and 4, e.g. 41-441, where 1 is for fully clear, 4 full-tint, with 2 and 3 for the light- and mid-tint states respectively. The ordering follows the numbering of the zones. Thus, for the label 41-441, zones 5, 7 and 8 are set to full- 


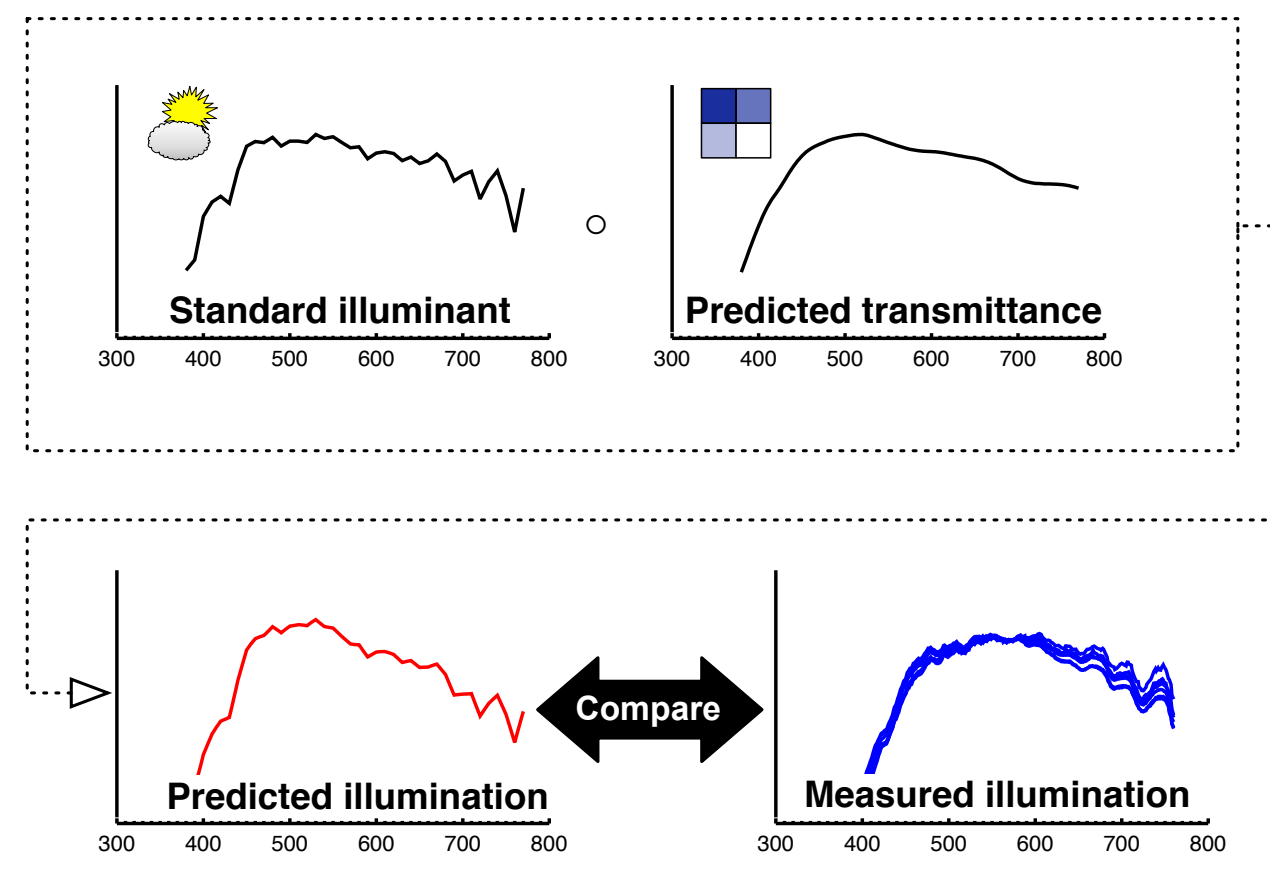

Figure 5: Illumination spectrum derived from predicted transmittance spectrum and standard illuminant - for comparison with measured illumination spectra.

tint (i.e. five panels altogether), and zones 6 and 9 are set to full clear (i.e. three panels altogether). The ratio vector $\mathbf{R}$ for this combination is therefore $\left[\begin{array}{llll}3 & 0 & 0 & 5\end{array}\right]$. Alongside the zoning graphic is a sketch showing the approximate positions of the four occupants and their typical view directions (A-D) whilst working. View $\mathrm{E}$ is a general view from the back of the room looking towards the glazing, and $\mathrm{F}$ is the view from the window wall towards the back of the room. Illumination spectra were measured at each of these six view points/directions for every combination of glazing state described below. To avoid contamination of the spectra, all electric lighting and computer monitors were switched off.

The aim for the validation was to test the predictions across a broad range of glazing states, and not to restrict the cases examined to those shown in Figure 3 - several of which were not achievable in the EC office due to the practicalities of the zoning regime for a 'live' installation (Figure 7). Illumination spectra in the room were measured under the six combinations of EC glass state listed in Table 1. After each change of glazing state we allowed for 15 minutes to ensure that the glazing had fully transitioned, a process which usually takes 5 to 10 minutes to complete.

\subsection{Measurement and normalisation of the spectra}

Spectra were measured using an MK350 handheld spectrometer produced by UPRtek. The spectra cover the range 360 to $760 \mathrm{~nm}$ and are output as normalised curves (peak equals 1). The sensor has an approximate cosine response, and so the spectra recorded are equivalent to spectral irradiance by a device that measures absolute units. The measured spectra are therefore also similar to what would be received by the eye when located at the various measurement positions and view directions indicated in Figure 7 . The MK350 also records illuminance and various derived quantities including correlated colour temperature (CCT) and colour rendering index (CRI). Tests showed that repeatability was 


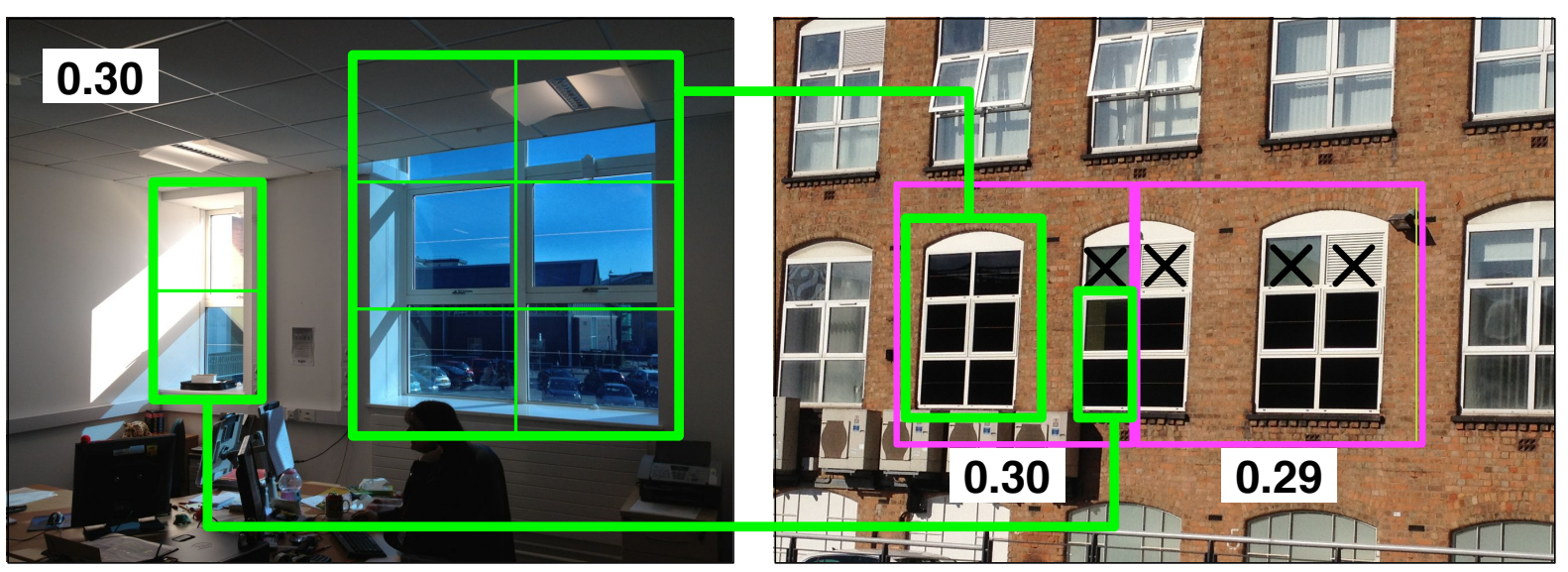

Figure 6: Photograph of Room 0.30 and the external facade.

\begin{tabular}{|c|c|c|c|c|c|}
\hline $\begin{array}{c}\text { Zone/state } \\
\text { label }\end{array}$ & {$\left[\begin{array}{ll}N_{62} & N_{20} \\
\end{array}\right.$} & $\left.\begin{array}{l}\mathbf{R} \\
N_{06} \\
N_{02}\end{array}\right]$ & $\begin{array}{l}\text { Measured } \\
C C T_{\text {mean }}\end{array}$ & $\begin{array}{l}\text { Measured } \\
C R I_{\text {mean }}\end{array}$ & $D_{55} \stackrel{r_{\text {mean }}}{\left(D_{65}, D_{75}\right)}$ \\
\hline $21-221$ & {$\left[\begin{array}{ll}3 & 5\end{array}\right.$} & $\left.\begin{array}{ll}0 & 0\end{array}\right]$ & $5211 \mathrm{~K}$ & 92 & $0.972(0.875,0.760)$ \\
\hline $31-331$ & {$\left[\begin{array}{ll}3 & 0\end{array}\right.$} & $\left.\begin{array}{ll}5 & 0\end{array}\right]$ & $5243 \mathrm{~K}$ & 93 & $0.977(0.902,0.782)$ \\
\hline $41-441$ & {$\left[\begin{array}{ll}3 & 0\end{array}\right.$} & $\left.\begin{array}{ll}0 & 5\end{array}\right]$ & $4970 \mathrm{~K}$ & 93 & $0.980(0.919,0.794)$ \\
\hline $41-444$ & {$\left[\begin{array}{ll}1 & 0\end{array}\right.$} & $\left.\begin{array}{ll}0 & 7\end{array}\right]$ & $4800 \mathrm{~K}$ & 94 & $0.906(0.700,0.514)$ \\
\hline $42-442$ & {$\left[\begin{array}{ll}0 & 3\end{array}\right.$} & $\left.\begin{array}{ll}0 & 5\end{array}\right]$ & $6845 \mathrm{~K}$ & 87 & $0.971(0.917,0.862)$ \\
\hline $43-443$ & {$\left[\begin{array}{ll}0 & 0\end{array}\right.$} & $\left.\begin{array}{ll}3 & 5\end{array}\right]$ & $11557 \mathrm{~K}$ & 84 & $0.974(0.947,0.916)$ \\
\hline
\end{tabular}

Table 1: Nomenclature for the measured states and a summary of the results.

very good and there was no practical advantage in taking multiple spectra at individual measurement points - an important consideration since we did not want the sun position to change significantly during each set of measurements. Comparison of daylight and artificial light spectra measured simultaneously with a 'laboratory grade' spectrometer (PhotoResearch 655) showed very good agreement.

The measurements were taken under sunny, clear sky conditions on a weekend day in order to not disrupt the normal occupants. The conditions were very stable with an almost total absence of clouds. The vertical illuminance recorded by the facade sensor (part of the EC control system installed by SAGE) shows the stability of the sky conditions on that day - the measurements were taken between $\sim 11$ am to $\sim 1 \mathrm{pm}$, Figure 8 . The sun azimuth was almost normal to the facade during the measurement period, and the sun was overwhelmingly the dominant source of illumination in the office space under those conditions. Thus, standard illuminant $D_{55}$ was chosen as the source used in Equation (9) to predict the illumination spectrum for the office space.

Predicted and measured spectra were normalised to $V(\lambda)$ such that the area under the normalised curve was the same for each, i.e. each of the spectra would produce the same illuminance. Thus any variation in the absolute levels of illumination (e.g. due to changing sun position) was not a factor in this evaluation, provided of course that the illumination 

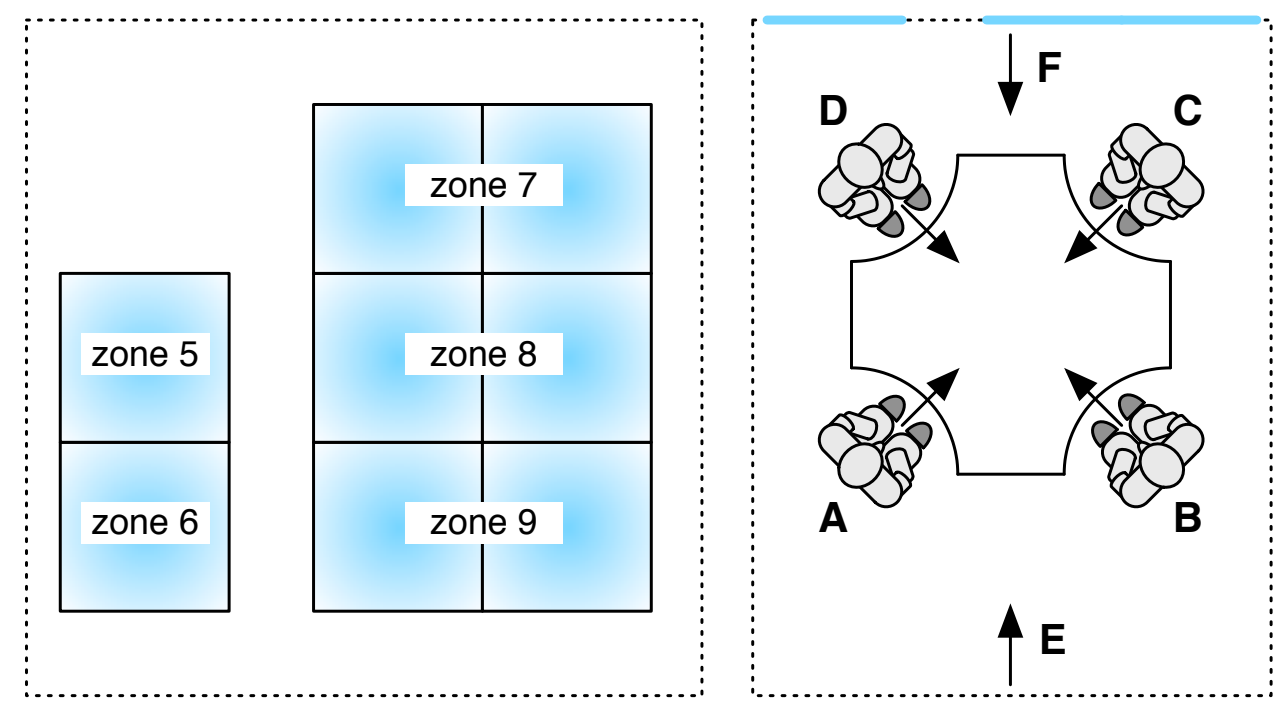

Figure 7: Zoning of the EC glass in Room 0.30 (compare with interior view in Figure 6) and a schematic (not to scale) of the office layout showing the occupant workstation positions and the six view directions $(\mathrm{A}-\mathrm{F})$ at which spectra were measured.

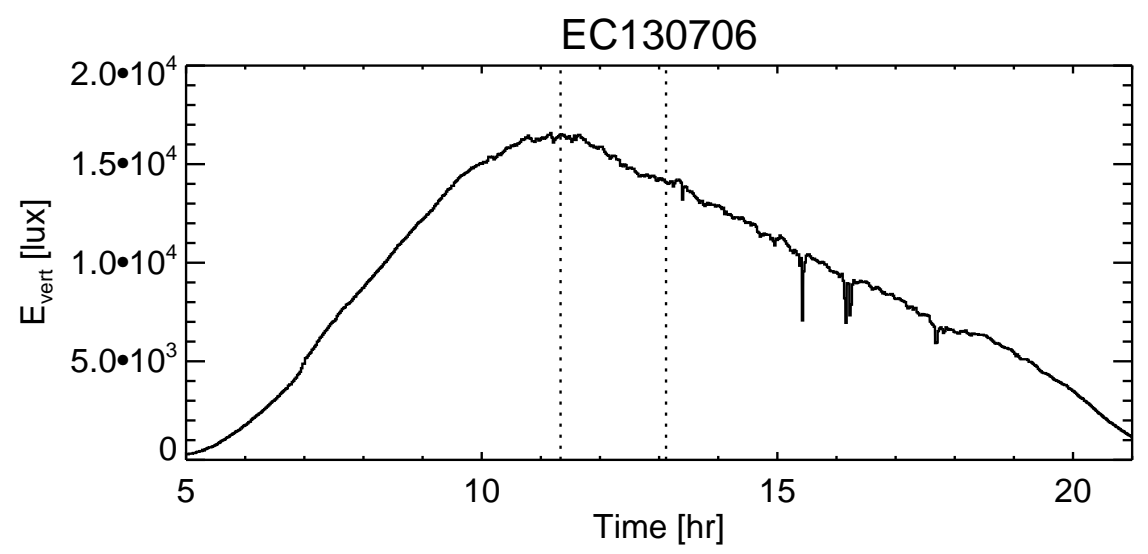

Figure 8: Vertical illuminance measured by the (external) facade sensor

spectrum remained constant during each set of measurements, which we believed was the case.

\subsection{Results}

Spectra measured at the six view points/directions shown in Figure 7 were compared to the theoretical illumination derived using Equations (7) and (9) for the six combinations of EC state given in Table 1. The set of six comparisons are shown in Figure 9. The predicted illumination spectrum is shown in red (dashed lines) and the six measured spectra in blue. Additionally, the visual sensitivity curve $V(\lambda)$ is shown in green (normalised to peak equals 1). Qualitatively, the agreement seems remarkably good with moderate divergence between theory and measured spectra for only one of the cases (41-444). For the four cases with at least one of the panels set to clear, the spectra appear fairly neutral with no pronounced shift to the blue. For comparison, eight daylight spectra measured in spaces with standard clear glazing are shown in the Appendix, Figure 10. 
The measured correlated colour temperature (CCT) and colour rendering index (CRI) further support the appearance of neutrality. Subjectively, at the time of measurement the illumination in the space appeared effectively neutral for these four cases. For the two cases without any panels set to clear the measured (and predicted) spectra show a pronounced peak in the blue part of the spectrum, i.e. around $470-480 \mathrm{~nm}$ and 450 $470 \mathrm{~nm}$ for, respectively, cases 42-442 and 43-443. The measured CCT and CRI for these two cases showed commensurate deviations from perceived neutrality. This corresponded also with subjective assessments at the time of measurement that the illumination in the space now had a noticeable grey or blue hue. Note also the conspicuous appearance of noise in the spectra measured for case 43-443. The effective visible transmittance for this combination was $3 \%$, i.e. $T_{v i s}=0.03$ - see annotation on each of the plots in Figure 9 . Thus the absolute levels of illumination measured at A-F were low: ranging from 11 to 43 lux. Such a combination of glazing state is unlikely to ever occur in an actual installation, and was chosen here solely for the purpose of testing the theoretical model.

The theoretical model assumes of course that the resulting illumination spectrum is the spatially homogeneous product of spectra from individual glazing panels in various states, i.e. the light is 'perfectly mixed'. In reality of course there will be some spatial variation in the illumination spectra dependent on such factors as:

- The configuration, zoning and state of the EC panels.

- The layout of the room and the surface reflectance properties.

- The sun position and patterns of direct illumination in the room.

Notwithstanding these considerations, the six spectra taken at the various points around the room show greater similarity than was first anticipated. The arrangement which might be expected to result in the most spatially inhomogeneous illumination spectrum was 41-444, i.e. 1 clear panel (zone 6) with the other seven panels at full-tint. In fact, the spread in the curves for 41-444 is no greater than for the other five cases, and actually appears to have less spread than most. We believe this is because the (high-angle) direct sun was reflected up off the white window sill, and subsequent reflections would be off the walls and ceiling - both white. This arrangement would appear to be particularly effective in 'mixing' the illumination spectra. This observation has implications for glazing/facade design with electrochromic glass where both neutrality of illumination and a high degree of daylight/solar control are required.

Quantitative comparison was made by determining the Pearson correlation coefficient $(r)$ between the theoretical illumination spectrum and each of the six measured spectra. Since the individual measured spectra were very similar, we present just the mean for the six values $\left(r_{\text {mean }}\right)$, Figure 9 and Table 1 . For all but 41-444, the agreement is very good, i.e. the $r_{\text {mean }}$ was greater than 0.97. For $41-444$ the $r_{\text {mean }}$ was 0.906 , noticeably lower than the other cases but still good.

It was instructive to test the effect of other standard daylight illuminants on the determined correlations. Daylight illuminants $D_{65}$ and $D_{75}$ can be taken to approximate, respectively, daylight from a sky without noticeable hue (e.g. overcast) and daylight from a moderate blue sky [9]. The $r_{\text {mean }}$ with the illumination spectrum now predicted using illuminants $D_{65}$ and $D_{75}$ are given (in parentheses) in Table 1. The correlations with the measured spectra are markedly less good, and, as expected, the illumination spectrum predicted using $D_{75}$ shows poorer agreement than that predicted using $D_{65}$. This trend 
further supports the hypothesis that the spectrum of illumination incident on the window was better represented by $D_{55}$ than either of the two other illuminants.

Note - the theory described in Section 2.1 has general applicability to any glazing type(s) of known spectral transmittance. However, the specifics of the predicted performance (e.g. Figures 3 and 4) are applicable only to the SageGlass ${ }^{\circledR}$ glazing manufactured by SAGE Electrochromics, Inc. as described by the IGDB files shown in Figure 2.
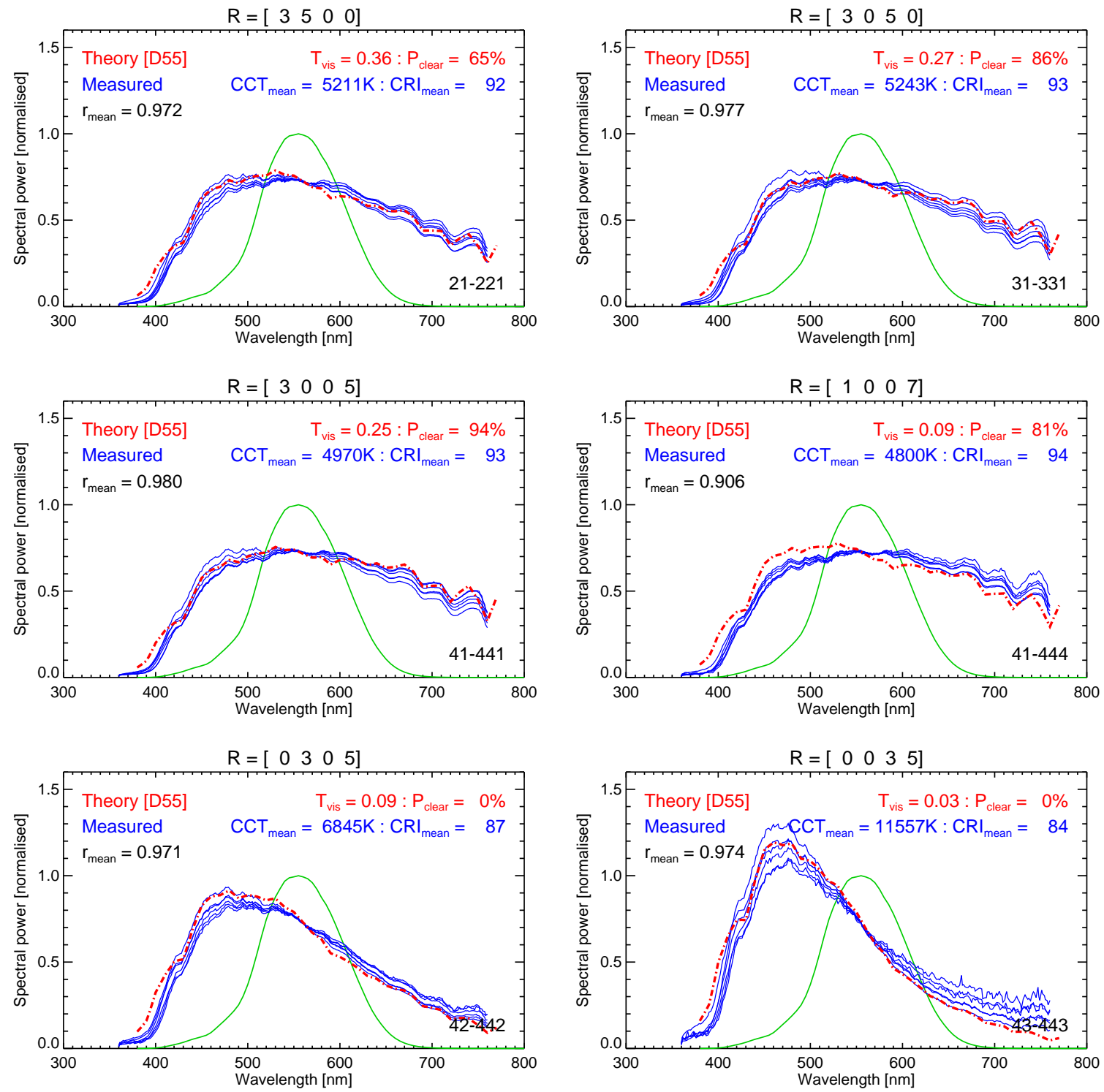

Figure 9: Predicted illumination spectrum and measured spectra for six combinations of clear and tinted EC glass.

\section{Discussion}

A theoretical model giving the spatially homogeneous spectral transmission curve for a combination of clear and tinted glass in arbitrary proportions has been presented. When combined with an applied daylight illuminant the model gives the predicted (spatially homogeneous) illumination spectrum for any particular combination of glazing. The model 
was tested against measurements of daylight spectra in an office with electrochromic glazing for six combinations of clear and tinted glass. Agreement between theory and measurement was good, perhaps remarkably so, and we consider the model to be validated for practical application in real world settings.

Facade design with EC glass should encourage good mixing of the light, though in practical terms this really means effective redistribution of daylight - especially direct sun - through glass which is clear. This can be achieved by positioning reflecting surfaces such as sills close to sections of the EC glass that are likely to be kept clear during normal operation. Additionally, some designs might benefit if the zoning were staged to vary throughout the day in order to maximise the reflection and redistribution of direct light through clear panels. Of course, a mixture of standard clear and EC glazing would be equally effective, provided there was certainty that the reduced flexibility did not diminish overall, annual performance, i.e. that users would never require any moderation of the light through the (standard) clear glass panels.

If light through the full-tint glass is not effectively mixed relative to light through clear panels (e.g. 'lost' through absorption by low reflectance surfaces), then the actual illumination spectrum is likely to be 'better' than that predicted by the model - though the effect may be slight. This may explain the small discrepancy between the theoretical illumination spectrum and the measured spectra for 41-444 where the illumination was indeed marginally more neutral than that predicted by the model.

The work described here suggests that the previously reported lower approval ratings for fixed-tint 'blue' glass compared to neutral or 'warm' tint glass [7] are not directly applicable to variable transmission glazing when some of the glass area is left in the clear state. Recall that the measured spectrum is that which would be received by the eye at the various positions in the room (Figure 7). This leads to an intriguing question: if the illumination spectrum received at the eye is effectively neutral, does it matter for the occupant what colour the windows appear to be? It is not possible to give a definitive answer to this question given the complexities of the relation between illumination and view for ordinary windows [10], let alone for variable transmission glass. However, one can offer some reasoned conjecture to this end. Two such speculations follow. The reader will note that the hypotheses outlined in the section below are solely for the purpose of promoting discussion - they will need to be tested using a variety of experiments involving human participants.

\subsection{Hypotheses regarding an observer's experience of tinted glass}

The subjects' expectation may have played a part in the assessment of the reported lower approval for fixed-tint 'blue' glass [7]. Few would claim that a view of blue sky through a window is unpleasant in any significant way, provided there are no visual discomfort issues related to direct sun etc. So it seems unlikely that we would have evolved to somehow have an in-built antipathy to 'blue views', at least for those which we perceive as natural. The light from the bluest part of a clear blue sky has a spectrum that is markedly shifted to the blue, with a CCT often greater than $10,000 \mathrm{~K}$ or even $15,000 \mathrm{~K}$. A blue sky however will, for an observer, always be associated with sunlight in some way. Even if sun illuminated surfaces are not directly visible, the illumination spectrum received at the eye would most likely contain a significant contribution from the ambient sunlight. But, on an overcast day, a grey sky seen through fixed-tint glass with a blue hue would not evoke the same response as the view of an actual blue sky (either through clear glass or directly through an open window). Firstly, we would not expect to see blue sky 
on a grey day (notwithstanding the fact that it is unlikely that EC glazing would be set to tint under such conditions). So, the spectral content of the view would conflict with our (preexisting) knowledge of the external daylight conditions. The tint would also make a dull sky appear even duller, thereby exacerbating any sense of drabness regarding the illumination. Perhaps just as important, if not more so, is the spectrum of illumination received at the eye. This would be very different from what we expect when there is a view of 'blue' through the window because, on a grey day, there would be no component of ambient (i.e. reflected) sunlight in the illumination spectrum received at the eye. Based on the findings reported here, it seems reasonable to speculate that a view containing panels of blue tinted EC glass may be perfectly acceptable to occupants - on sunny days - provided that the illumination spectrum contains a significant component of sunlight, i.e. a small proportion of the panels are set to clear. This is because:

(a) we have an 'in-built' expectation that blue in the distant view through windows is associated with the presence of sunlight in the illumination spectrum received at the eye; and,

(b) the daylight through the clear EC glass dominates the resulting illumination spectrum, giving a natural appearance and good colour rendering.

For the second speculation the reader is asked to consider a hypothetical scenario where the occupant, initially, has their back to the window. At workstation positions $\mathrm{C}$, $\mathrm{D}$ and also at $\mathrm{F}$ looking to the back of the room, the measured illumination spectra (for the cases with one or more clear panels) were sufficiently neutral that an observer might not be aware of the tint state of the glass, unless of course they had a direct view of the window. Furthermore, there was no significant difference in spectra between these and the other three view points which did have sight of the windows. Now consider a scenario where very similar illumination conditions - both for the spectrum and absolute (i.e. lux) values - were achieved using either (i) standard glass with blinds or (ii) EC glazing in a combination of clear and full-tint panels. Would an occupant, with their back to the windows, be able to notice any significant difference between these two scenarios? One would think not if the spectra were similar. When the occupant turns to face the windows, the illumination conditions (spectrum and lux values) may again be very similar, though of course the views will be completely different. With standard glass/blinds the view to the outside may be completely blocked, prohibiting the occupant from relaxing their focus on a distant scene. With EC glazing a clear view is preserved even when at full-tint - a feature that occupants may find preferable to standard blinds which act like a 'shutter'.

To summarise the above: what is seen/experienced by the occupant needs to be congruent with what is known/expected regarding the external conditions.

\subsection{Additional considerations}

An anonymous reviewer raised a number of discussion points that are outside of the scope of the main topic of this paper. However, they are interesting points worthy of further discussion/investigation - two are briefly touched upon in this section.

The Kruithof curve has been cited in numerous publications since it first appeared in 1941 [11]. The curve suggests that lower CCT light (i.e. a 'warm' hue) is preferred for low illumination levels, and high CCT (i.e. a 'blue' hue) at high illumination levels. This would appear at first glance to be in 'opposition' to what occurs in a space with EC glazing. Firstly, it has been pointed out by a number of authors that the boundaries of the Kruithof curve have, in the majority of cases, not been successfully reproduced in 
subsequent tests. In a 1990 paper by Boyce and Cuttle, the authors state that the "results obtained show quite clearly that, once the subject is fully adapted to the conditions, the CCT of good colour rendering lamps in the range $2700 \mathrm{~K}$ to $6300 \mathrm{~K}$ has little effect on people's impressions of the lighting of the room" [12]. However, more significantly for EC glazing, the study reported here shows that daylight illumination spectra can be kept well within the range considered normal by ensuring that just a relatively small proportion of the EC glazing is held in the clear state.

If used effectively, light redirecting shades can reprocess a portion of the direct sunlight into diffuse daylight than could then enhance the overall daylight levels in the space. In contrast, EC glazing will reduce overall light levels whenever the glazing tints. A consequence of maintaining a clear view whatever the state of of tint is that EC glazing cannot redirect light. Of course, this is so for all materials that have purely specular transmission, e.g. essentially all architectural glazing intended to provide the possibility of a clear view out. EC glazing certainly has the potential to greatly diminish the overall daylight levels in a space: by up to a factor of 60 for the latest SAGE product. It is rarely likely to be the case that occupants would wish such a large reduction in overall daylight levels, as opposed to those experiencing direct sun. However, the work reported here demonstrates that some EC glazing should, in the main, always remain in the clear state. A 'rule-of-thumb' for EC control could be to always maximise the proportion of glazing held in the clear state whilst ensuring that visual and/or thermal discomfort is minimised. Although the emphasis for this paper was on maintaining a neutral daylight spectrum, the work described here is being developed to investigate absolute levels of daylight illumination in addition to spectrum using lighting simulation techniques.

\subsection{Future work}

Findings from the case-study evaluation of user acceptance for the two EC offices will, we hope, help to answer some of the questions posed above. Occupant feedback related to the perception of colour in the EC offices is being collated as part of the regular program of data collection [8]. At the time or writing, the authors are preparing a number of experiments to more rigorously assess the subjective perception of colour in the EC offices under normal operation.

More generally, this investigation has shown that it is possible to carry out fairly exacting measurements of daylight spectra in real buildings under normal conditions. Recent models to predict the non-visual effects of daylight have incorporated the spectral properties of the different standard daylight illuminants [9]. Thus it is timely to demonstrate that the field measurement of daylight spectra in buildings is now a practical possibility, for both general investigations of the internal luminous environment and also for the testing of theoretical models.

\section{Funding}

The EC glazing in the two offices was installed by SAGE Electrochromics, Inc., and Ms. Kelly Waskett is supported by a De Montfort University PhD Studentship. However, no other grant or commercial funding was received for the work reported in this article.

\section{Acknowledgements}

Prof. Mardaljevic acknowledges the support of Loughborough University, and Dr. Painter the support of De Montfort University. Dr. Ivan Korolija (De Montfort University) assisted with the measurement of the spectra in the EC office. 


\section{References}

[1] Roger J. Mortimer. Switching colors with electricity. American Scientist, 101(1):3845, 2013.

[2] Ruben Baetens, Bjorn Petter Jelle, and Arild Gustavsen. Properties, requirements and possibilities of smart windows for dynamic daylight and solar energy control in buildings: A state-of-the-art review. Solar Energy Materials and Solar Cells, 94(2):87-105, 22010.

[3] H. Sanders and A. Andreau. The next generation of dynamic glazing product performance. Proceedings of Glass Performance Days, Tampere, Finland. 13-15 June, 2013.

[4] S. Selkowitz, M. Rubin, E. S. Lee, and R. Sullivan. A review of electrochromic window performance factors. SPIE International Symposium on Optical Materials Technology for Energy Efficiency and Solar Energy Conversion XIII, Freiburg, Germany, 1994.

[5] F. Gugliermetti and F. Bisegna. Visual and energy management of electrochromic windows in mediterranean climate. Building and Environment, 38(3):479-492, 2003.

[6] E. S. Lee, E. S. Claybaugh, and M. LaFrance. End user impacts of automated electrochromic windows in a pilot retrofit application. Energy and Buildings, 47(0):267-284, 42012 .

[7] H. Arsenault, M. Hébert, and M-C. Dubois. Effects of glazing colour type on perception of daylight quality, arousal, and switch-on patterns of electric light in office rooms. Building and Environment, 56:223-231, 102012.

[8] R. Kelly, B. Painter, J. Mardaljevic, and K. Irvine. Capturing the user experience of electrochromic glazing in an open plan office. CIE Midterm conference - Towards a new century of Light, Paris, France 12-19 April, 2013.

[9] J Mardaljevic, M Andersen, N Roy, and J Christoffersen. A framework for predicting the non-visual effects of daylight - Part II: The simulation model. Lighting Research and Technology, 46(4):388-406, 2014.

[10] J. A. Veitch and A. Galasiu. The Physiological and Psychological Effects of Windows, Daylight, and View at Home: Review and Research Agenda. National Research Council of Canada, Institute for Research in Construction, NRC-IRC RR-325, 2012.

[11] A. A. Kruithof. Tubular luminescence lamps for general illumination. Philips Technical Review, 6(3):65 - 73, 1941.

[12] P. R. Boyce and C. Cuttle. Effect of correlated colour temperature on the perception of interiors and colour discrimination performance. Lighting Research and Technology, 22(1):19-36, 031990. 


\section{Appendix}

\section{A Measured Daylight Spectra for Standard Clear Glazing}

Daylight spectra were measured in a number of office and teaching spaces with standard clear glazing. There was no attempt to create a 'representative' sample that characterises the range of what occupants might describe as a space with 'typical' daylight illumination. Such a study, as far as the authors are aware, has not yet been carried out. Indeed, it is not yet clear what, in a strict quantifiable sense, constitutes 'typical' daylight illumination. Consequently, the spectra presented in this section are solely to illustrate something of the range that can be encountered in daylit spaces with standard clear glazing. The spaces were semi-randomly selected on the basis of access and location - the various buildings were close by to each other on the De Montfort University campus (Leicester, UK). Each plot contains a photograph of the approximate field of view as 'seen' by the spectrometer.

Measurements were taken with the artificial lighting switched off and well away from any (switched on) computer displays whose luminous output could 'contaminate' the daylight spectra. The absence of any obvious spikes in the spectra suggests that no significant artificial light contribution was present at the time of measurement. The spaces had standard clear double glazing with no obvious tint, colour or applied film. However, the windows were not closely examined, so the presence of an inconspicuous solar control film cannot be ruled out. Measured spectra for eight spaces are shown in Figure 10. The measured CCTs range from $4232 \mathrm{~K}$ to $7080 \mathrm{~K}$. There are conspicuous differences between the various spectra resulting from a number of possible causes. These could be any of the following acting singly or in combination:

- Relative contributions of sunlight and skylight to the space.

- Sunlight reflecting off surfaces that have a saturated hue, e.g. blue carpet.

- Effective enhancement of skylight contribution from specular reflections off 'shiny' desk surfaces (this could be the cause of the high CCT of $7080 \mathrm{~K}$ for one of the spectra).

Notwithstanding these evident differences in the spectra, there is no reason to believe that users of these spaces would perceive the daylight quality as somehow untypical of that which would be expected for natural illumination from standard clear glazing. 

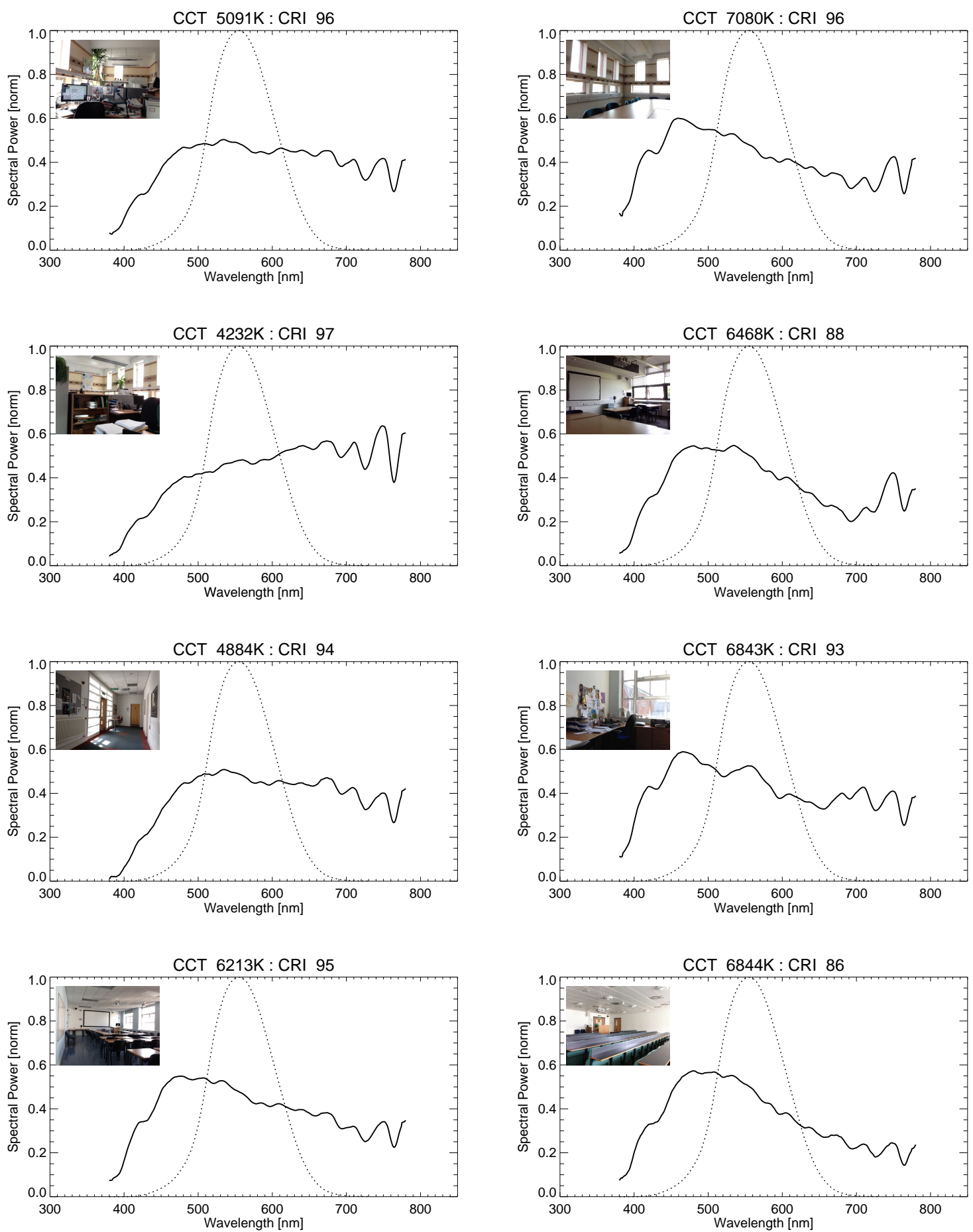

Figure 10: Measured daylight spectra in spaces with standard clear glazing. 